\title{
Do instantons like a colorful background?
}

\author{
H. Gies ${ }^{1, a}$, J. Jaeckel ${ }^{2, b}$, J.M. Pawlowski ${ }^{1, c}$, C. Wetterich ${ }^{1, d}$ \\ ${ }^{1}$ Institut fuer Theoretische Physik, Philosophenweg 16, 69120 Heidelberg, Germany \\ 2 Deutsches Elektronen-Synchrotron DESY, Notkestrasse 85, 22607 Hamburg, Germany
}

Received: 20 September 2006 /

Published online: 19 January 2007 - @ S Springer-Verlag / Società Italiana di Fisica 2007

\begin{abstract}
We investigate chiral symmetry breaking and color symmetry breaking in QCD. The effective potential of the corresponding scalar condensates is discussed in the presence of non-perturbative contributions from the semiclassical one-instanton sector. We concentrate on a color singlet scalar background which can describe chiral condensation, as well as a color octet scalar background which can generate mass for the gluons. Whereas a non-vanishing singlet chiral field is favored by the instantons, we have found no indication for a preference of color octet backgrounds.
\end{abstract}

\section{Introduction}

Instantons, being pseudo-particles associated with tunneling processes, generate genuine non-perturbative effects in QCD. In the seminal work of 't Hooft $[1,2]$ it was realized that they mediate an effective interaction between (light) quarks [1-9]. This "instanton interaction" is attractive in the color singlet channel; hence, instantons presumably play a role in the mechanism of chiral symmetry breaking [10-12]. In addition, they also provide for an interaction in color octet channels or in color triplet and sextet "diquark" channels. Mean-field computations based on a point-like instanton interaction have been employed as a central tool for investigations of color superconductivity at high baryon density [13-17], or for a description of the baryon and meson spectrum and interactions in the vacuum in a Higgs picture with spontaneous color symmetry breaking $[8,18,19]$. This phenomenologically quite successful scenario requires a quark-anti-quark condensate in the color octet channel, giving rise to the question as to whether instantons support quark condensation in this channel.

Symmetry breaking by a condensation phenomenon requires an interaction that lowers the free energy if condensates are formed. The bosonic condensates can be quark bilinears or even higher-order composites. In the case of instantons, a rich interaction structure is indeed provided: for $N_{\mathrm{f}}$ light quarks, instantons typically induce an interaction between $2 N_{\mathrm{f}}$ quark fields, which can be paired in many ways. This is one of the reasons why instantoninduced multi-fermion interactions have often been used

\footnotetext{
a e-mail: H.Gies@thphys.uni-heidelberg.de

b e-mail: jjaeckel@mail.desy.de

c e-mail: J.Pawlowski@thphys.uni-heidelberg.de

d e-mail: C.Wetterich@thphys.uni-heidelberg.de
}

as a starting point for investigations in the mean-field approximation. However, in the approximation of a point-like multi-fermion interaction, mean-field theory is ambiguous: by means of a Fierz transformation, the quarks can be grouped in different ways. For example, products involving color non-singlet Lorentz scalars can be exchanged by products of color singlets in vector or tensor representations of the Lorentz group and vice versa. In view of this ambiguity, the relative strength between color octet and singlet channels remains undetermined, since the color octet channels can be completely removed or enhanced by suitable re-orderings [20]. Similar problems arise for the other colored channels used in the high-density computations.

For further progress towards reliable computations, the Fierz ambiguity of the mean-field computation has to be resolved. This can be done in different ways. A first possibility explicitly includes the fluctuations of composite bosons after partial bosonization. Then, the dependence on the particular choice of bosonization (Fierz ambiguity) gets substantially reduced, as demonstrated by functional renormalization group techniques [21]. A second approach attempts to resolve the ambiguity by explicitly taking the momentum dependence of the instanton-induced vertex into account. Finally, we propose a third method in this article that avoids altogether the use of the multi-fermion vertex and rather computes directly the instanton contribution to the free energy in the presence of selected condensates. The various approaches have different strengths and shortcomings, and a reliable picture will probably only emerge by a combination of them.

The advantage of a study of the momentum dependence of the instanton-induced vertex is based on the observation that pole-like structures which arise from the effective exchange of quark-anti-quark or quark-quark bound states can be associated to the given channel of 
the bound state. In contrast to a point-like interaction, such pole structures can no longer be moved to another channel by Fierz reordering. Momentum-dependent vertex functions can be dealt with using functional methods, such as Dyson-Schwinger equations, NPI effective actions, functional renormalization group (RG) or suitable combinations. In particular, we envisage the functional $R G$ as a promising approach towards a quantitative study for the condensation phenomena at hand, for reviews see $[22-25]$. The computation of the flow equations involves only a narrow momentum range around a given renormalization scale $k$, thus reducing the impact of an incomplete knowledge of the detailed momentum dependence of the full propagators and vertices; see, e.g., [25-29]. Its application to the present problem requires an implementation for the non-perturbative sector of gauge theories, see e.g. [22, 26-28, 30-32], also employing bosonization techniques as developed in $[21,25,33-35]$, or NPI- and NPPI-flows as discussed in $[24,25,36,37]$. In particular, the Fierz-type ambiguity of the present problem can be resolved within a 2PPI-effective-action approach, since all possible (local) fermionic pairings are effectively taken into account by this approach [20].

In this work, we consider a more direct approach to instanton-induced color symmetry breaking by taking advantage of the following observation: possible condensates can be viewed as background fields that are coupled to quarks and gluons via Yukawa and gauge interactions. We concentrate here on scalar color singlet and octet condensates. In the presence of a singlet condensate, all three light quarks become massive, thus influencing the weight of the fermion determinant in the instanton calculation. Additional octet condensates induce a mass split between an octet of fermions (here associated with the baryon octet) and a singlet. Furthermore, all gluons acquire mass through the Higgs mechanism. Both effects modify the instanton contribution to the free energy. In particular, the effective condensate-dependent gluon mass acts as an effective infrared cutoff, strongly suppressing the contribution of instantons of size larger than the inverse gluon mass. Furthermore, the infrared cutoff stops the running of the gauge coupling such that the gauge coupling remains small for sufficiently large octet condensates, and perturbation theory becomes applicable. By computing the instanton contribution to the free energy in presence of the condensates, we get access to those parts of the effective potential that violate the axial $U(1)_{\text {A }}$ symmetry. Under the hypothesis that these parts dominate the octet dependence of the potential, we may try to draw conclusions if the minimum occurs for vanishing or non-vanishing octet condensate. Our computation of this response is based on two theoretical concepts: on the one hand, the full functional integral is evaluated in the semiclassical oneinstanton approximation. On the other hand, the decoupling of massive modes is taken care of by a proper threshold behavior of the running coupling, as it is suggested by the functional RG.

Our method needs assumptions how the non-vanishing singlet and octet condensates influence the masses of quarks and gluons. In practice, this is done by an ansatz for the effective action which describes the couplings of quarks and gluons to the color singlet and octet condensates. Apart from the restrictions imposed by color and flavor symmetry, the details of this effective action are not known. This is one of the most severe restrictions on the quantitative reliability of our computation. Nevertheless, the qualitative features of mass generation for quarks and gluons can be captured in a simple picture. We consider here a local interaction with low powers of the condensates, in particular the chiral color singlet scalar $\sigma_{a b}$ with flavor indices $a, b, \ldots$ and the color octet scalar $\chi_{a b, i j}$ with nontrivial structure for flavor and color $(i, j, \ldots)$. Our ansatz for the interactions between the condensate fields, quarks and gluons can be summarized in the following Euclidean effective Lagrangian [19],

$$
\begin{aligned}
\mathcal{L}= & \mathrm{i} Z_{\psi} \bar{\psi}_{i} D_{i j} \psi_{j}+\frac{1}{2} F_{i j}^{\mu \nu} F_{j i, \mu \nu} \\
& +Z_{\chi} \operatorname{tr}\left\{\left(D^{\mu} \chi\right)_{i j}^{\dagger}\left(D^{\mu} \chi\right)_{i j}\right\}+Z_{\sigma} \operatorname{tr}\left\{\partial^{\mu} \sigma^{\dagger} \partial^{\mu} \sigma\right\} \\
- & \mathrm{i} Z_{\psi} \bar{\psi}_{i}\left[\left(h \sigma \delta_{i j}+\tilde{h} \chi_{i j}\right) \frac{1+\gamma^{5}}{2}\right. \\
& \left.+\left(h \sigma^{\dagger} \delta_{i j}+\tilde{h} \chi_{j i}^{\dagger}\right) \frac{1-\gamma^{5}}{2}\right] \psi_{j} \\
& +U_{0}(\sigma, \chi) .
\end{aligned}
$$

Here, we have included all power-counting relevant and marginal interaction operators as well as an effective potential for the background fields for completeness. In (1), we treat $\sigma$ and $\chi_{i j}$ as $3 \times 3$ matrices in flavor space and contract over the flavor indices of the quarks. A successful phenomenology of QCD based on an effective Lagrangian of this form has been worked out in $[18,19,39]$.

Obviously, the reliability of our conclusions will depend on whether the ansatz (1) gives a qualitatively correct picture for the response to non-vanishing condensates. We therefore present a few additional arguments for its motivation. Associating the condensates $\sigma, \chi$ with corresponding fermion composites $\sim \bar{\psi} \psi$, the interactions of the type specified in (1) arise naturally from fundamental QCD, as can be studied with techniques developed in $[21,33-35,37]$. In particular, box diagrams of the type shown in Fig. 1 play an important role. In this work, we choose the viewpoint that these effective interactions are present in the dominant momentum region for the instanton contribution, being generated by $U(1)_{\mathrm{A}}$-preserving interactions, also partly at higher momentum scales. We do not attempt here to compute the parameters appearing in the effective action (1) except for the gauge coupling. For a qualitative study, we treat the Yukawa couplings $h, \tilde{h}$ as well as the wave function renormalization factors $Z_{\psi}, Z_{\sigma}$, $Z_{\chi}$ as free parameters.

In the presence of non-vanishing background fields $\sigma$, chiral symmetry is broken, whereas $\chi$ acts like the Higgs scalar, giving masses to gluons and quarks. For the present purpose, it suffices to investigate in detail the following two directions in field space:

$$
\sigma_{a b}=\sigma \delta_{a b}, \quad \chi_{a b, i j}=\frac{1}{\sqrt{6}} \chi\left(\delta_{i a} \delta_{j b}-\frac{1}{3} \delta_{i j} \delta_{a b}\right) .
$$



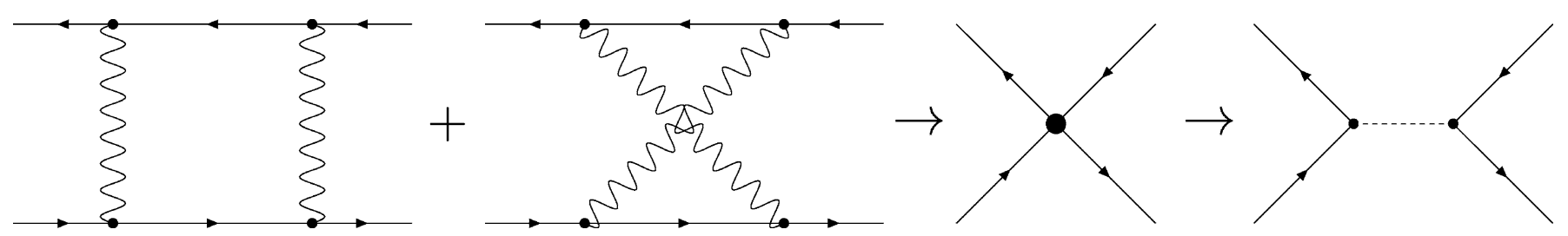

Fig. 1. Box diagrams with fundamental QCD interactions (left two diagrams) generate effective (non-local) four fermion interactions (middle). Using rebosonization [33] these can be translated to (approximately local) Yukawa interactions interactions with propagating composite bosons (right)

These configurations correspond to the condensates of standard chiral symmetry breaking and a color-flavor locked [38] combination of quarks and anti-quarks, respectively. In this background, all fermions aquire mass,

$$
M_{1}=h \sigma+\frac{8}{3 \sqrt{6}} \tilde{h} \chi, \quad M_{8}=h \sigma-\frac{1}{3 \sqrt{6}} \tilde{h} \chi,
$$

with a split between the octet mass $M_{8}$ and the singlet mass $M_{1}$ for $\chi \neq 0$. The fermion determinant in the instanton contribution depends only on $M_{8}$ and $M_{1}$. We use the freedom of scaling of the fields $\sigma$ and $\chi$ to set $h=\tilde{h}=1$. In this normalization, $\sigma$ and $\chi$ are directly related to the masses. In the Higgs picture of the QCD vacuum, the expectation value for $M_{8}$ should be associated with the mass of the lowest baryon octet and $M_{1}$ with a baryon singlet, possibly $\Lambda(1405)$, yielding [40] $M_{8}=1.15 \mathrm{GeV}$, $M_{1}=-1.4 \mathrm{GeV}$ or $\sigma_{0}=866 \mathrm{MeV}, \chi_{0}=-2.08 \mathrm{GeV}$. In our approach, we treat $\sigma$ and $\chi$ as free variables. The octet condensate in (3) provides for an equal mass for all eight gluons,

$$
M_{g}=Z_{\chi}^{1 / 2} g|\chi|
$$

Here, $g$ is the renormalized coupling taken at an appropriate scale. The $\chi$ dependence of $g$ will be discussed in detail below. Then $Z_{\chi}$ remains the only undetermined parameter of our ansatz. The phenomenological ansatz of $[19,40]$ associates $M_{g}$ with the average mass of the lowest spin-one meson octet, $M_{g} \simeq 850 \mathrm{MeV}$ and suggests $Z_{\chi}^{1 / 2} \simeq 1 / 15$.

The paper is organized as follows. In Sect. 2 , we discuss the various effects of the quark and gluon masses on the instanton integral. In the subsequent section, Sect. 3, we discuss the asymptotic behavior of the instanton contribution to the free energy. In Sect. 4, we investigate which condensate backgrounds are preferred by the instantons. Our conclusions are presented in Sect. 5 .

\section{Effective potential in one-instanton approximation}

Consider a given background of scalar fields $\sigma$ and $\chi$, as introduced above. Our aim is to compute the instanton contribution to the effective potential for $\sigma$ and $\chi$ in the presence of fluctuating quarks and gluons. For homogeneous $\sigma$ and $\chi$, the effective action $\Gamma$ thus decomposes into

$$
\begin{aligned}
\Gamma[\sigma, \chi] & \equiv \Omega U(\sigma, \chi) \\
& =\Omega\left(U_{0}(\sigma, \chi)+U_{\text {inst }}(\sigma, \chi)+U_{\text {inst }}^{*}(\sigma, \chi)\right),
\end{aligned}
$$

where $\Omega$ denotes the spacetime volume. The non-anomalous contribution $U_{0}(\sigma, \chi)$ conserves the axial $U(1)_{\mathrm{A}}$ symmetry and will not be computed here. The anomalous contribution $U_{\text {inst }}$ is induced by configurations with nontrivial topology, mediating also $U(1)_{\mathrm{A}}$ violation [41]. We determine this part in a semiclassical approximation based on instanton methods. In particular, we resort to the approximation of a gas of dilute instantons in which $U_{\text {inst }}$ can be expressed by an integral over the instanton size $\rho$ and the product of gluonic (ghosts included) and fermionic fluctuation determinants in a one-instanton background (see Appendix A),

$$
U_{\text {inst }}=-\frac{1}{\Omega} \int_{0}^{\infty} \mathrm{d} \rho \exp \left(-8 \pi^{2} / g^{2}(\rho)\right) \Delta_{\mathrm{gl}}(\chi, \rho) \operatorname{det} M_{\psi, i j} .
$$

Here, the exponential factor reflects the classical action of the instanton, and $\Delta_{\mathrm{gl}}$ summarizes the contributions from gluons and ghosts in the instanton background. The last factor, with

$$
M_{\psi, i j}=-\not D_{i j}+\sigma \delta_{i j}+\chi_{i j}
$$

represents the fermion determinant which is of central interest to our work. In particular, it contains the zero modes of the Dirac operator which are responsible for anomalous contributions and give rise to a strong $\sigma$ and $\chi$ dependence even for small values of these fields.

It is useful to decompose (6) into a factor $\zeta_{z}(\sigma, \chi, \rho)$ arising from the fermionic zero modes, and another nonzero-mode factor $\zeta_{\mathrm{n}}(\sigma, \chi, \rho)$ that summarizes all remaining (classical, gluonic, fermionic) contributions:

$$
U_{\text {inst }}(\sigma, \chi)=-\int \mathrm{d} \rho \zeta_{\mathrm{n}}(\sigma, \chi, \rho) \zeta_{\mathrm{z}}(\sigma, \chi, \rho) .
$$

All contributions have been studied frequently in the literature, beginning with the seminal work of ' $t$ Hooft $[1,2]$. As important new aspects, we include the color octet scalar and take the threshold behavior due to decoupling of massive modes into account. 


\subsection{Lowest order in the background fields}

Assuming that $\sigma$ and $\chi$ are small compared to all other scales, their main influence arises from the zero-mode contribution. In particular, the non-zero-mode factor $\zeta_{\mathrm{n}}$ does not depend on the scalar fields to lowest order. For an $S U\left(N_{\mathrm{c}}\right)$ gauge theory with $N_{\mathrm{f}}$ flavors, $\zeta_{\mathrm{n}}$ reads $^{1}[1,2]$

$$
\zeta_{\mathrm{n}}(\rho)=D_{\mathrm{S}} \rho^{-5}\left(\frac{8 \pi^{2}}{g^{2}(\rho)}\right)^{2 N_{\mathrm{c}}} \exp \left(-\frac{8 \pi^{2}}{g^{2}(\rho)}\right) .
$$

Here, $D_{\mathrm{S}}$ is a scheme-dependent constant. A discussion of the scheme dependence including the difference between massive and massless regularization schemes can be found in Appendix B. Our scheme has been motivated by the functional $\mathrm{RG}$ which generically provides for mass-dependent schemes that automatically account for a proper decoupling of massive modes. This is a convenient feature of our RG-inspired scheme; however, we observe no qualitative scheme dependencies of our results. For example, to zeroth order in the fields, $D_{\mathrm{S}}$ in our RG regularization scheme is given by (see $[41,43,44]$ and Appendix C)

$$
\begin{aligned}
D_{\overline{\mathrm{RG}}}=D_{\overline{\mathrm{MS}}}= & \frac{2 \exp \left(\frac{5}{6}-i g\right)}{\pi^{2}\left(N_{\mathrm{c}}-1\right) !\left(N_{\mathrm{c}}-2\right) !} \\
& \times \exp \left(-1.51137 N_{\mathrm{c}}+0.29175 N_{\mathrm{f}}\right) \\
= & 6.005 \times 10^{-3},
\end{aligned}
$$

where the last equality holds for $N_{\mathrm{c}}=N_{\mathrm{f}}=3$.

As discussed in the Appendices B and $\mathrm{C}$, our $\overline{\mathrm{RG}}$ scheme is constructed such that it matches the $\overline{\mathrm{MS}}$ scheme in the small mass limit. It was demonstrated in [45] that the $\overline{\mathrm{MS}}$ scheme gives satisfactory agreement with lattice data in the ultraviolet. Without a color-flavor mixing mass matrix $(\chi=0)$, the eigenmodes of $\not D$ are also eigenmodes of $M_{\psi}$ and we are led to $[1,2,7]$

$$
\begin{aligned}
\zeta_{\mathrm{z}}(\rho, \sigma, \chi) & =\left\langle\operatorname{det}_{\text {flavor }}\left\langle\psi_{0}(a, i)\left|M_{\psi, i j}\right| \psi_{0}(b, j)\right\rangle\right\rangle_{S U(3)} \\
& =:-\rho^{N_{\mathrm{f}}} V(\sigma, \chi),
\end{aligned}
$$

where the inner angled brackets denote the scalar product of the zero modes $\psi_{0}$, and the outer angled brackets denote a group average over all possible directions for the instanton in color space. In the last step, we have separated off the simple $\rho$ dependence $\sim \rho^{N_{\mathrm{f}}}$ and defined the auxiliary potential $V(\sigma, \chi)$. We have also used the persistence of (quasi-) zero modes in the presence of the regularization [7]. For $\chi \neq 0, \not D$ and $M_{\psi}$ do not commute in general, e.g. for condensates $\chi$ with (2). The eigenmodes of $\not D$ and $M_{\psi}$ do not agree anymore for $\chi \neq 0$. Therefore, strictly speaking, (11) does not hold in general. However, in leading order of an expansion in $\chi$ and $\sigma$ it holds true, as shown in Appendix D. Inserting these findings into (8), we obtain

$$
U_{\text {inst }}(\sigma, \chi)=V(\sigma, \chi) \int \mathrm{d} \rho \rho^{N_{\mathrm{f}}} \zeta_{\mathrm{n}}(\rho)=: \zeta V(\sigma, \chi) .
$$

\footnotetext{
1 In Appendix C, we briefly review the contributions from the zero and the non-zero modes starting from results given in $[1,2,42]$. Moreover, we use this appendix to introduce our regularization scheme.
}

For $N_{\mathrm{f}}<4, \zeta$ is a finite number for physically admissible running couplings from the UV to the IR as discussed in Appendix E. For small $\sigma$ and $\chi$, the potential $V(\sigma, \chi)$ carries all dependence on the scalar condensates.

So far our discussion has made no use of a specific color or flavor structure for the background fields. Let us now specialize to the condensates specified in (2). Using the gauge-group averages computed in [3] we find (see Appendix $\mathrm{D}$ for details)

$$
\begin{aligned}
V(\sigma, \chi) & =-\sigma^{3}+\frac{1}{72} \sigma \chi^{2}+\frac{1}{648 \sqrt{6}} \chi^{3} \\
& =-\left(\sigma+\frac{1}{6 \sqrt{6}} \chi\right)^{2}\left(\sigma-\frac{1}{3 \sqrt{6}} \chi\right) .
\end{aligned}
$$

In this crude approximation where $U(\sigma, \chi)=\zeta V(\sigma, \chi)$ with $\zeta$ being a field-independent constant, we observe two flat directions, $\sigma=-\frac{1}{6 \sqrt{6}} \chi$ and $\sigma=\frac{1}{3 \sqrt{6}} \chi$, but no global minimum. In fact, $V(\sigma, \chi)$ is unbounded from below, similar to the findings in [20]. In the present case, this simply signals the breakdown of the approximation of small $\sigma$ and $\chi$.

Let us assume for a moment that the potential becomes stable beyond this approximation or by the inclusion of $U_{0}(\sigma, \chi)$ (cf. (5)). Then one might speculate that the first flat direction, $\sigma=-\frac{1}{6 \sqrt{6}} \chi$, which is a line of local minima for $\sigma<0$, characterizes a global minimum (the second flat direction is not even a local minimum). However, in this case, the ratio $r=\left|\frac{\sigma}{\chi}\right|=\frac{1}{6 \sqrt{6}} \approx 0.068$ is far from the phenomenologically reasonable range $r \sim 0.4$ [40]. Since $V$ is completely determined by the zero modes of the massless Dirac operator, this flat direction will not be lifted by the inclusion of higher order corrections in the bosonic fields in the one-instanton approximation, as long as the split into zero- and non-zero-mode parts remains justified. A similar flat direction was also found in $[20]$.

Let us furthermore assume that, for instance, $U_{0}$ induces a non-zero VEV for $\sigma$. Since $V(\sigma, \chi)$ prefers a positive $\sigma$, the resulting potential $V(\sigma, \chi)$ in the $\chi$ direction looks like the solid line sketched in Fig. 2. The case of no color octet condensate, $\chi=0$, then is a local minimum. For larger $\chi$, the higher-order corrections from the nonzero-mode contribution and the threshold effects will set in, stabilizing the potential in the $\chi$ direction. Now it is a dynamical question as to whether this stabilization sets in early, i.e., for rather small $\chi$, such that no other minimum is induced (dotted line). Or stabilization could only modify the region of large $\chi$ (dashed) line, such that the $\chi^{3}$ term of (13) wins out in between, and induce a color octet condensate.

The second scenario of color octet condensate formation seems more difficult to be realized, since the $\sim \sigma^{3}$ term and the $\sim \sigma \chi^{2}$ one are of opposite sign and the coefficient of the $\chi^{3}$ term is rather small. Unfortunately, the small coefficients in front of $\sigma \chi^{2}$ and $\chi^{3}$ in the potential (13) will limit even the qualitative reliability of our investigation. As an effect of the color averaging, the po- 


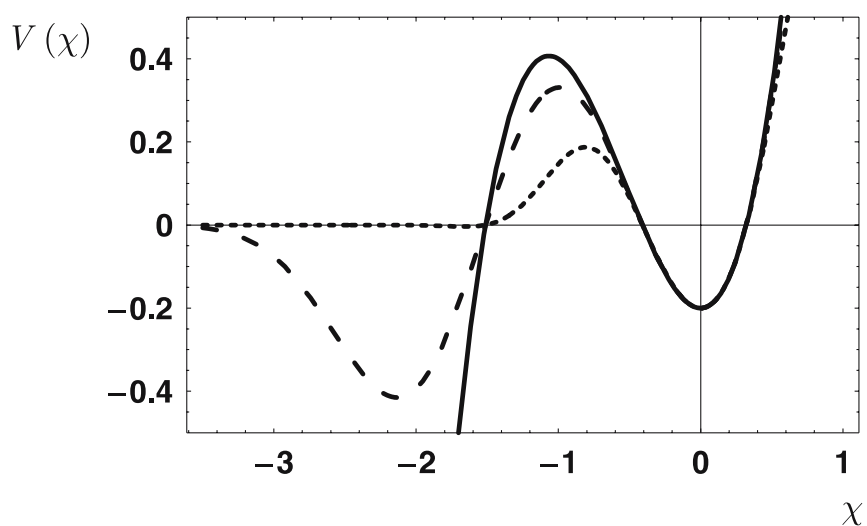

Fig. 2. Schematic plot of $V(\sigma, \chi)$ at fixed $\sigma>0$. Without higher-order corrections, the potential is unbounded from below (solid line) with a local minimum at $\chi=0$. If the cutoff mechanism provided by the higher-order corrections is strong (dotted line) the global minimum remains at $\chi=0$. However, if the suppression sets in only at rather large values of $\chi$ (dashed line) we have a global minimum at $\chi \neq 0$ in addition to a local one at $\chi=0$

tential in the $\chi$ direction is almost flat for a given value of $\sigma$, in contrast to the pronounced potential in the $\sigma$ direction. For a given $\sigma$, the weak dependence of $V$ on the "direction" $\chi / \sigma$ could easily be overwhelmed by corrections in higher orders in $\sigma$ and $\chi$ that are much more difficult to control. Despite this caveat, a quantitative analysis remains interesting and will be presented in the next sections.

Let us close this lowest-order consideration with the remark that $\sigma$ and $\chi$, in general, are complex fields. However, complex field values typically lead to large $C P$ violation, making them phenomenologically unacceptable; this is the reason why we restricted our analysis to real field values. If a non-trivial phase between the octet and singlet condensates is favored in case of non-vanishing $|\chi|$, this may lead to an argument against the formation of color octet condensates in general. In order to demonstrate this point we assume for a moment that the effective potential for the relative phase between $\chi$ and $\sigma$ is dominated by the small field instanton contribution $U \approx \zeta V(\sigma, \chi)$. Then, real positive values of $\sigma$ would be preferred due to the instanton contribution. This would in turn lead to a positive "mass term" $\sim \chi^{2}$ (cf. Fig. 5b), originating from the $\sigma \chi^{2}$ term in (13); for imaginary $\chi=\mathrm{i}|\chi|$, this turns into $-\sigma|\chi|^{2}$. Combining this with the small $\chi^{3}$ term, the relative minimum of $V(\sigma, \chi)$ for fixed $\sigma>0,|\chi|>0$ would occur for a complex $C P$ violating $\chi$. Unfortunately, the impact of this observation is weakened by the very small coefficients of the $\sigma \chi^{2}$ and $\chi^{3}$ terms arising in our approximation. The approximate flatness in the $\chi$ direction makes the potential influence of the other effects large. In this context we observe that the $U(1)_{\mathrm{A}}$-conserving part $U_{0}$ in (5) also contributes to the effective potential for the phase between $\chi$ and $\sigma$, for example with terms $\sim \sigma^{* 2} \chi^{2}+$ c.c. Only the common phase of $\chi$ and $\sigma$ is protected by the $U(1)_{\mathrm{A}^{-}}$ symmetry and is uniquely determined by the instanton part.

\subsection{Beyond small condensates}

As demonstrated in the preceding section, the instantoninduced effective potential can, in principle, support a mechanism for spontaneous color octet condensation. Whether or not this mechanism is realized, however, requires a study that is valid for larger values of $\sigma$ and $\chi$. The consequences of large condensates are twofold. First, the fermion masses are no longer small. This affects the non-zero-mode contribution $\zeta_{\mathrm{n}}$ as well as the running of the gauge coupling. Also a mixing between zero modes and non-zero modes is induced. Second, a color non-singlet field gives an effective mass to the gauge fields, which again modifies the running of the gauge coupling (now the pure gauge contribution). In addition, it provides for an effective infrared cutoff for the $\rho$ integration.

\subsubsection{Effects on the running gauge coupling}

Fermion and effective gauge boson masses exert an immediate influence on the running of the gauge coupling. For momenta smaller than the mass of a given quark or gluon degree of freedom, the corresponding fluctuations of this degree of freedom are suppressed. As a consequence, these fluctuations do no longer contribute to the running of the coupling. This decoupling of massive modes can directly be implemented in the $\beta$ function for the running coupling, which we write as

$$
\begin{aligned}
\partial_{t} g^{2} & \equiv k \frac{\mathrm{d}}{\mathrm{d} k} g^{2} \\
& =-\frac{1}{8 \pi^{2}} g^{4}\left(\frac{11}{3} N_{\mathrm{c}} l_{g}\left(\frac{M_{g}^{2}}{k^{2}}\right)-\frac{2}{3} N_{\mathrm{f}} l_{f}\left(\frac{\left|M_{8}\right|^{2}}{k^{2}}\right)\right), \\
t & \equiv \ln \frac{k}{\Lambda},
\end{aligned}
$$

where $M_{g}$ and $M_{8}$ are the gluon and the octet masses given in (3) and (4), and $k$ denotes an RG momentum scale. The threshold functions $l_{g, f}(x)$ approach unity for small argument, $l_{g, f}(0)=1$, corresponding to the fact that the physical or effective masses play no role in the UV limit $k \rightarrow \infty$. For large argument, i.e., for momentum scales $k$ below a given mass, the threshold functions drop to zero rapidly, $l_{g, f}(x \gg 1) \rightarrow 0$, which implements the decoupling of massive modes from the renormalization flow. The threshold functions are not universal but regularization scheme dependent. For generic mass-dependent schemes, the threshold functions interpolate smoothly between the two limits. ${ }^{2}$ For the explicit computations, we set the threshold functions equal, $l_{g}(x)=l_{f}(x)=l(x)$, and use

$$
l(x)=\frac{1}{(1+x)^{3}} .
$$

\footnotetext{
2 For mass-independent schemes such as the $\overline{\mathrm{MS}}$ scheme, threshold functions do not appear directly; but in order to describe the physics above and below a mass threshold adequately, theories with a correspondingly different particle content have to be matched at the mass threshold. This can equally be described by an effective threshold function which changes its slope discontinuously at a mass threshold.
} 
This is a typical form for a threshold function, occurring in calculations based on the functional RG. Of course, the one-loop form used in (14) only serves as an example. A similar analysis of mass threshold behavior applies to any loop order and even fully non-perturbatively. We would like to stress that it is this threshold behavior where the additional free parameter $Z_{\chi}$ enters via $M_{g}$; cf. (4).

As a result of this decoupling mechanism, the effective running coupling is now field dependent, $g(k, \sigma, \chi)$. Inserting this into (9) and (12) results in an additional field dependence of the effective potential. Qualitatively, the gauge boson mass weakens the increase of the gauge coupling. Owing to the exponential of the classical action $\sim \exp \left(-8 \pi^{2} / g^{2}\right)$ in (9), this leads to a total suppression of the instanton contribution. The fermion threshold behavior has the opposite effect due to the minus sign in the $\beta$ function, reflecting their charge-screening nature.

\subsubsection{Effect on the instanton determinant}

The condensates give masses to fermions and gluons; hence the corresponding fluctuation determinants have to be evaluated for this massive case. Let us first consider the massive fermion determinant, i.e., the non-vanishing shift of the fermionic (non-zero) eigenmodes due to the effective fermion mass (3). This problem has been solved recently using an efficient method to perform the mode sum [46, 47]. The result interpolates smoothly between the analytically known small- and large-mass expansions [48, 49]. These calculations have been performed with a color singlet quark mass $m$ in the MS scheme. Here, we neglect the difference in the effect of the singlet and octet quark mass and approximate

$$
m=\frac{1}{3} \sqrt{\left|M_{1}\right|^{2}+8\left|M_{8}\right|^{2}} .
$$

For our purposes, we have to adapt the results of $[46,47,49]$ to our massive $\mathrm{RG}$ regularization scheme, as derived in Appendix $\mathrm{C}$, and use the following interpolating function:

$$
\begin{aligned}
N_{\mathrm{f}} K(x) & :=\left.\ln \operatorname{det}^{\prime}(-\not D+m)\right|_{\mathrm{RG}} \\
& =-\frac{2}{3} N_{\mathrm{f}}\left(H(x)+\frac{3}{4}\right)+\ln _{\left.\operatorname{det}^{\prime}(-\not D+m)\right|_{\mathrm{MS}}} \\
& \simeq N_{\mathrm{f}}\left[-\ln (x)-a_{1}+\frac{\ln (x)+a_{1}-a_{2} x^{2}-a_{3} x^{4}}{1+a_{4} x^{2}+a_{5} x^{4}+a_{6} x^{6}}\right],
\end{aligned}
$$

with $x=\rho m$. The function $H(x)$ is defined in (C.9), and

$$
\begin{array}{lll}
a_{1}=0.792, & a_{2}=3.58, & a_{3}=0.0842, \\
a_{4}=0.00115, & a_{5}=23.5, & a_{6}=9.28 .
\end{array}
$$

The primed determinant det' in (17) is for the space of non-zero modes. As shown in Fig. 3, this function interpolates smoothly between the small- and large-mass regimes.

A similar behavior as for the fermion determinant is expected for the non-vanishing gluon mass in the gluon determinant. However, this effect is sub-leading, the dominant effect being the modification of the classical action at the minimum, see e.g. $[1,2,7]$ : for constant $\chi$, this gives

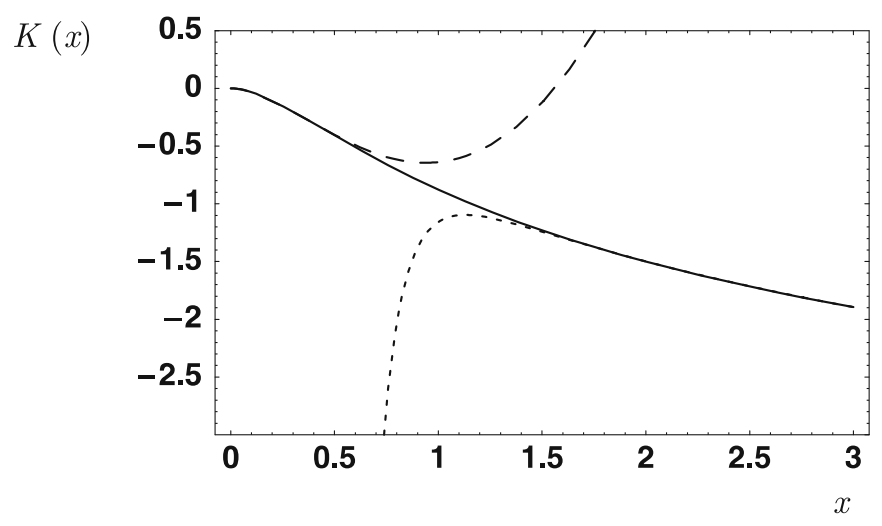

Fig. 3. The solid line gives the interpolating function $K(x)$, smoothly connecting the small mass (dashed) and the large mass approximations [46, 47, 49]

a contribution to the classical action $\Delta S_{\mathrm{cl}}=-6 \pi^{2} Z_{\chi}|\chi|^{2} \rho^{2}$ and therefore a factor of (cf. (6))

$$
\begin{aligned}
& \exp \left(-8 \pi^{2} / g^{2}(\rho)\right) \Delta_{\mathrm{gl}}(\chi, \rho) \\
& \quad \rightarrow \exp \left(-8 \pi^{2} / g^{2}(\rho)-6 \pi^{2} Z_{\chi}|\chi|^{2} \rho^{2}\right) \Delta_{\mathrm{gl}}(\rho)
\end{aligned}
$$

in the integral (12). To summarize, the full inclusion of $\sigma$ and $\chi$ in the fermion determinant and the Higgs-type of contribution to the classical action result in our final formula for the effective potential $\left(N_{\mathrm{c}}=N_{\mathrm{f}}=3\right)$ :

$$
\begin{aligned}
U_{\text {inst }}= & D_{\overline{\mathrm{RG}}} V(\sigma, \chi) \int \mathrm{d} \rho \rho^{-2}\left(\frac{8 \pi^{2}}{g^{2}(\rho)}\right)^{6} \\
& \times \exp \left(-\frac{8 \pi^{2}}{g^{2}(\rho)}-6 \pi^{2} Z_{\chi}|\chi|^{2} \rho^{2}+3 K(m \rho)\right),
\end{aligned}
$$

where $K$ is given in (17), and $D_{\overline{\mathrm{RG}}}$ is defined in (10). Once the running of the gauge coupling is specified, e.g., using the one-loop form of (14) and identifying the RG scale with the inverse instanton radius, $k=1 / \rho$, we can investigate the landscape of the instanton-induced contribution to the effective potential for $\sigma$ and $\chi$. For fixed $\sigma$, an additional $\chi$ dependence arises from the explicit term $\sim|\chi|^{2}$ in the "classical part", the dependence of $g(\rho)$ on $\chi$ and the threshold effect $K(m \rho)$. Our approximation of $K(m \rho)$ reflects probably only poorly the dependence on the ratio $\chi / \sigma$, and we have also neglected the mixing between the fermionic zero modes and non-zero modes which would modify $V(\sigma, \chi)$.

\section{Asymptotic behavior of the effective potential}

In order to obtain a more analytic understanding of the effective potential $U_{\text {inst }}$, let us investigate its asymptotic behavior for the different regimes of small and large fields $\sigma$ and $\chi$. Of particular interest is the interplay between this asymptotic behavior and the running of the gauge coupling. As an important caveat, it should be kept in mind that our 
derivation of the effective potential is based on the semiclassical instanton-gas approximation. This approximation implicitly assumes that the one-instanton contribution is small, which translates into a small value of $U_{\text {inst }}$. Therefore, whenever a large asymptotic behavior of $U_{\text {inst }}$ is encountered, this may not necessarily reflect the true behavior but rather signal the breakdown of the instanton-gas approximation.

Our derivation of (19) so far made use of the specific oneloop running of the gauge coupling given in (14). Assuming that the functional dependence on the running coupling holds also in the general case, we use the form of (19) also for other theoretically or phenomenologically motivated running gauge couplings. For definiteness, we will use gauge couplings with the following infrared $(\rho \rightarrow \infty)$ properties:

$$
\begin{aligned}
& \left.g^{2}(\rho)\right|_{\rho \rightarrow \infty} \\
& \sim \begin{cases}\text { const, } & \text { fixed point, } \\
|\ln (\rho)|^{p_{\log },} & \text { logarithmic divergence, } \\
\rho^{p_{\text {power }}}, & \text { power law divergence, } \\
g_{\text {pert }}^{2}(\rho) \Theta\left(\Lambda_{\mathrm{QCD}}-\frac{1}{\rho}\right) & \text { perturbative } \\
+\Theta\left(\frac{1}{\rho}-\Lambda_{\mathrm{QCD}}\right) \infty, & \end{cases}
\end{aligned}
$$

with positive constants $p_{\log }$ and $p_{\text {power }}$; each infrared behavior will be adapted to show the same decoupling properties for massive modes as displayed in (14) and (15). For simplicity, we assume that this IR behavior does not depend on the number of fermions. These running couplings and the corresponding resulting instanton densities at vanishing external fields are shown in Fig. 7 in Appendix E. The effective potential for non-vanishing $\sigma$ and $\chi$ is strongly influenced by fermion and gauge boson mass effects, respectively. Hence, the effective potential is expected to behave differently in the various directions of the $\sigma, \chi$ plane. For definiteness, let us investigate four cases: (1) $\sigma$ small, $\chi=0$; (2) $\sigma \rightarrow \infty, \chi=0$; (3) $\sigma=0, \chi$ small and (4) $\sigma=0, \chi \rightarrow \infty$.

The $\rho$ integration can be performed analytically by splitting the integration domain into several intervals, each of them dominated by a different effect. For case (1), for instance, there is a UV regime $\left(0, \rho_{\text {pert }}\right)$ where the running coupling is well approximated by one-loop perturbation theory without the influence of mass thresholds. In the consecutive interval, $\left(\rho_{\text {pert }}, \frac{c}{\sigma}\right)$ (with $c$ being some $O(1)$ constant) the gauge coupling is dominated by non-perturbative dynamics, but fermion masses still do not play an important role. Finally, in the interval $\left(\frac{c}{\sigma}, \infty\right)$ the fermions are heavy, compared to the scale $1 / \rho$, and pure gluodynamics dominates the running coupling. Neglecting logarithmic dependencies on $\sigma$ or $\chi$, we find

$$
\begin{aligned}
& U_{\text {inst }}(\sigma, \chi) \\
& \sim \begin{cases}A_{1} \sigma^{N_{\mathrm{f}}}+B_{1} \sigma^{4+2 N_{\mathrm{c}} p_{\text {power }},} & (1): \sigma \text { small, } \chi=0, \\
A_{2} \sigma^{4-\beta_{0}}+B_{2} \sigma^{4\left(1-\frac{\beta_{0}}{\beta_{0}^{\prime}}\right)}, & (2): \sigma \rightarrow \infty, \chi=0, \\
A_{3} \chi^{N_{\mathrm{f}}}+B_{3} \chi^{4+\frac{4 N_{\mathrm{c}} p_{\text {power }}}{2+p_{\text {power }}},}, & (3): \sigma=0, \chi \text { small }, \\
A_{4} \chi^{4-\beta_{0}}, & (4): \sigma=0, \chi \rightarrow \infty,\end{cases}
\end{aligned}
$$

where all terms with coefficients $A_{i}$ arise from the perturbative interval $\rho \in\left(0, \rho_{\text {pert }}\right)$, and those with coefficients $B_{i}$ arise from the various non-perturbative intervals. Of course, only the $B_{i}$ terms depend on the form of the running coupling specified in (20); in fact, $B_{1}$ and $B_{3}$ vanish for the "perturbative" gauge coupling of (20). Furthermore, $\beta_{0}=\frac{11}{3} N_{\mathrm{c}}-\frac{2}{3} N_{\mathrm{f}}$ is the one-loop coefficient of the $\beta$ function with fermions, and $\beta_{0}^{\prime}=\frac{11 N_{\mathrm{c}}}{3}$ denotes the oneloop coefficient for pure gluodynamics. Not surprisingly, the logarithmically divergent and the fixed-point coupling yield the same results on this level of accuracy; hence the parameter $p_{\log }$ of (20) does not enter (21).

For $N_{\mathrm{f}}>4+2 N_{\mathrm{c}} p_{\text {power }}$, the small- $\sigma$ and small- $\chi$ behavior may significantly be modified compared to the perturbative expectation $\sigma^{N_{\mathrm{f}}}, \chi^{N_{\mathrm{f}}}$. Here the possible dependence on the infrared behavior of the gauge coupling appears to be important. However, this simply reflects the fact that naive IR convergence in the $\rho$ integration is lost for $N_{\mathrm{f}}>4$, as we have already noted before. In this case, the convergence is now restored by a combination of the suppression due to the finite fermion mass $\exp \left(-N_{\mathrm{f}} K(m \rho)\right)$ and the suppression from the gauge coupling $\left(\frac{8 \pi^{2}}{g^{2}(\rho)}\right)^{2 N_{\mathrm{c}}}$ at the expense of a direct dependence on the IR behavior of the gauge coupling.

In the large-field regime, the potential grows faster than $\sigma^{4}, \chi^{4}$ only if $\beta_{0}<0$ which corresponds to theories without asymptotic freedom. Conversely, for asymptotically free theories, the instanton-induced potential will not dominate over the non-anomalous part of the potential $U_{0}$ which can be expected to exhibit a $\sigma^{4}, \chi^{4}$ growth for reasons of universality. In view of the caveat mentioned in the beginning of this subsection, we interpret this result as a successful self-consistency check of the instanton-gas approximation. Furthermore, for $N_{\mathrm{c}}=N_{\mathrm{f}}=3$ and fixed $\sigma$, the instanton contribution vanishes by $U_{\text {inst }} \sim \chi^{-5}$ for large $\chi$. The instanton potential is therefore stabilized in the $\chi$ direction.

\section{Numerical analysis of the effective potential}

Guided by the analytic knowledge obtained so far for the effective potential, let us study our full result for the instanton-induced effective potential $U_{\text {inst }}(\sigma, \chi)$ obtained numerically from (19). For definiteness, we use - as an example - a one-loop form for the gauge coupling modified such that it approaches an IR fixed point at $g_{\mathrm{fix}}^{2}=100$ in the absence of condensates; this is in the ball park of IR results from RG flow equations [32]. Our conclusions remain similar for all other running couplings proposed in (20).

Let us first confirm the asymptotic behavior obtained analytically above along the $\sigma$ and $\chi$ axes. As is visible in Fig. 4a, displaying $U(\sigma, 0)$, the potential along the $\sigma$ axis is unbounded from below for $\sigma \rightarrow \infty$. In particular, for $N_{\mathrm{f}}=N_{\mathrm{c}}=3$, the resulting asymptotics of (21), yielding $|U(\sigma, 0)| \sim \sigma^{\frac{8}{11}}$, is confirmed. Let us stress that the overall sign of the potential is negative for positive $\sigma$, since the integral in (19) is always positive and the prefactor $V(\sigma, 0)=-\sigma^{3}$ is negative. From this, we draw two conclu- 


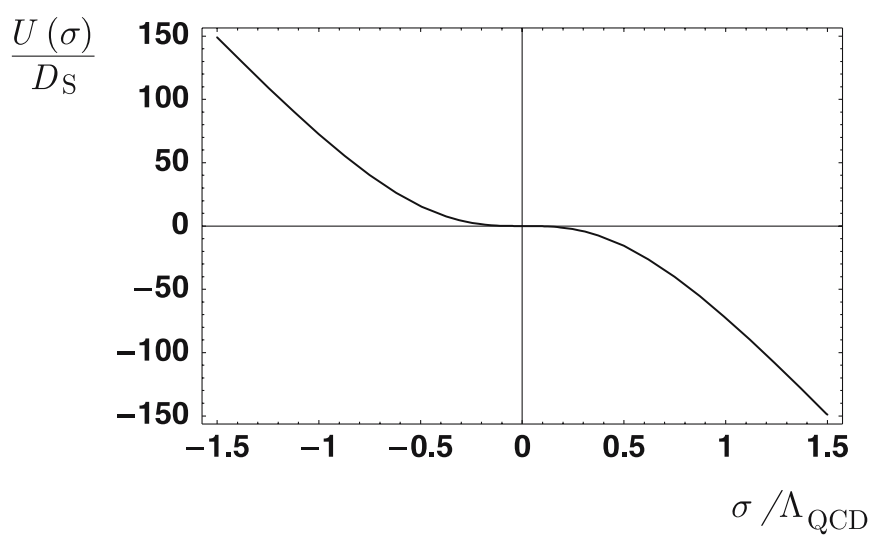

(a)

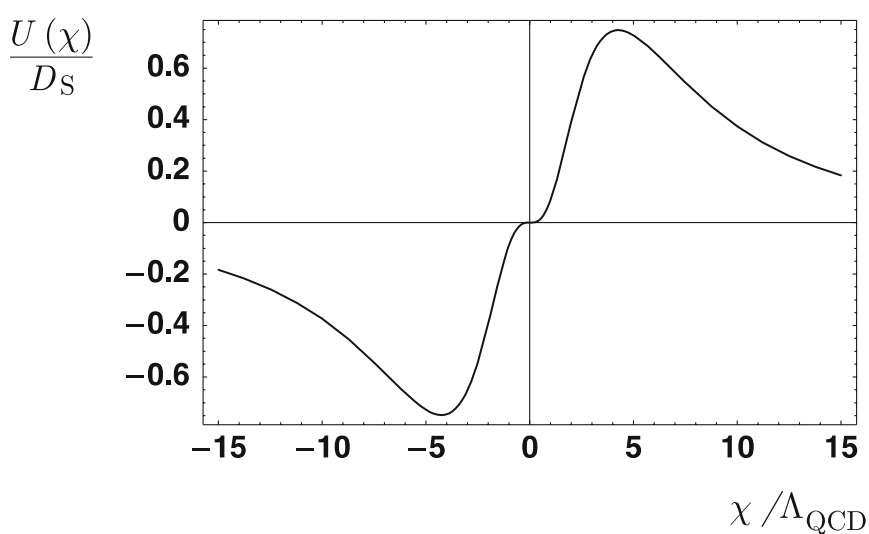

(b)

Fig. 4. Sections of the instanton potential $U_{\text {inst }}(\sigma, 0)$ a and $U_{\text {inst }}(0, \chi) \mathbf{b}$ in units of $\Lambda_{\mathrm{QCD}}$. The different large-field behavior is clearly visible: the potential in the positive $\sigma$ direction goes to $-\infty$, whereas it rapidly approaches 0 for large $|\chi|$. Note the different scales of the potential itself for the $\sigma$ and the $\chi$ direction. (Both plots use $Z_{\chi}^{1 / 2}=1 / 15$ and a gauge coupling approaching an IR fixed point $g_{\text {fix }}^{2}=100$. The renormalization-scheme-dependent constant $D_{\mathrm{S}}$ is scaled out.)

sions: first, the instanton potential favors chiral symmetry breaking, but the value of the condensate is not determined by the instanton potential alone (at least in our simple oneinstanton approximation). Second, the complete instanton potential $U_{\text {inst }}(\sigma, \chi)$ cannot have a global minimum, since there is a direction in which the potential always decreases.

Next we consider a pure $\chi$ field. Figure $4 \mathrm{~b}$ shows that the instanton potential becomes flat rather rapidly for large $\chi$, as expected from (21). Negative $\chi$ are clearly preferred. ${ }^{3}$ From Fig. 4 a, we can read off the location of the minimum: $\chi_{\min } \sim 4.26 \Lambda_{\mathrm{QCD}} \sim 1.4 \mathrm{GeV}$ for $\Lambda_{\mathrm{QCD}}=$ $330 \mathrm{MeV}$. This results in an encouraging $M_{g} \sim 350 \mathrm{MeV}$.

Nevertheless, it is important to observe that the potential in the $\chi$ direction is rather shallow compared to the $\sigma$ direction, the relative height being $\sim 10^{-3}$. This is a direct consequence of the relative prefactors in the potential $V(\sigma, \chi)$ of (13). The dependence of the absolute value of $\chi$ on our remaining free parameter $Z_{\chi}$ is strong, whereas it remains weak for $M_{g}$. This is in agreement with the expectation that, in the octet direction, the threshold effect of the gluon mass $M_{g}$ is much stronger than that of the quark masses $\sim \chi$. For our quantitative results, we use the value $Z_{\chi}^{1 / 2}=1 / 15$ which is in the phenomenologically acceptable range $[19,40]$.

Finally, let us study the complete potential depending on both fields $\sigma$ and $\chi$. Even though we have already observed that the instanton contribution alone does not have a global minimum, it is nevertheless worthwhile to look for a local one. Such a local minimum indeed exists at $(\sigma, \chi) \approx$ $(-0.27,-4.2) \Lambda_{\mathrm{QCD}}$ with the absolute depth of the potential being $\left.U(\sigma, \chi)\right|_{\text {loc. min. }} \approx-1.42 D_{\mathrm{S}} \approx 0.009$ in units of $\Lambda_{\mathrm{QCD}}$ (and using $D_{\overline{\mathrm{MS}}} \approx 6 \times 10^{-3}$ ). Since this minimum has a too small $|\sigma| \sim 90 \mathrm{MeV}$ and is extremely shallow, it

\footnotetext{
3 Let us stress that the relative sign of $\sigma$ and $\chi$ is indeed important, since it changes the parity of some particles in the spectrum of the model (1). Moreover, owing to the $U(1)_{\mathrm{A}}$ anomaly, it is not clear whether the sign in the Yukawa couplings can be rotated away by a chiral transformation.
}

is not physically acceptable. Any generic non-anomalous contribution $U_{0}$ is likely to remove this minimum.

Since the local minimum is not acceptable and a purely instanton-induced global minimum does not exist, let us redo our analysis with one additional assertion: we assume that the non-anomalous contribution $U_{0}(\sigma, \chi)$ to the effective potential supports scalar singlet condensation via spontaneous symmetry breaking for sufficiently strong gauge coupling (in fact, this has been shown to happen generically in QCD-like theories in [34]). In order to introduce as few parameters as possible, we simply assume that $U_{0}$ fixes a non-zero value of $\sigma$, leaving the detailed form of $U_{0}$ aside. Now, since $U_{0}$ is of non-anomalous origin, its form reflects the full chiral symmetry (even though it can exhibit a symmetry-breaking minimum), implying its invariance under $\sigma \rightarrow-\sigma$. Hence, $U_{0}$ does not prefer a particular sign of $\sigma$. By contrast, $U_{\text {inst }}$ does prefer positive values of $\sigma$ as displayed in Fig. 4a.

The instanton potential for small positive $\sigma$ is depicted in Fig. 5a. We observe that the global minimum for $\chi$ is non-vanishing only for very small values of $\sigma$. Beyond the critical value of $\sigma_{\text {crit }} \approx 0.128 \Lambda_{\mathrm{QCD}}<50 \mathrm{MeV}$ the global minimum is at $\chi=0$. This holds, in particular, for realistic values of $\sigma=1, \ldots, 4 \Lambda_{\mathrm{QCD}}$, which is plotted in Fig. 5b. For $\sigma \gtrsim \Lambda_{\mathrm{QCD}}$ the dominant feature of the $\chi$ dependence of $U_{\text {inst }}$ is simply the vanishing of $U_{\text {inst }}$ for large $\chi$ which results in a relative minimum at $\chi=0$. Figure $5 \mathrm{~b}$ summarizes one of our main results, namely, that the instanton-induced potential appears incapable of giving rise to an octet condensate in the present instanton-gas approximation.

Let us study the potential also for negative fixed $\sigma$. Even though positive values are clearly preferred by $U_{\text {inst }}$, observation only constrains the modulus of $\sigma$ to be in the realistic range $|\sigma| \sim 1, \ldots, 4$. A negative sigma indeed always give rise to a global minimum at $\chi \neq 0$, i.e. is supportive of a color octet; see Fig. 6. However, for realistic values of $\sigma$, the instanton-induced potential is extremely shallow again, such that this minimum is likely to be washed out 


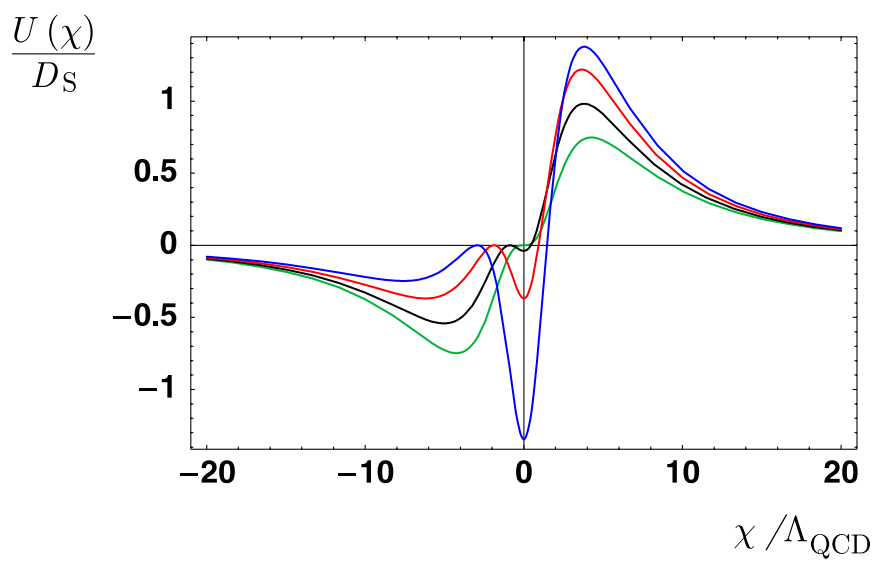

(a)

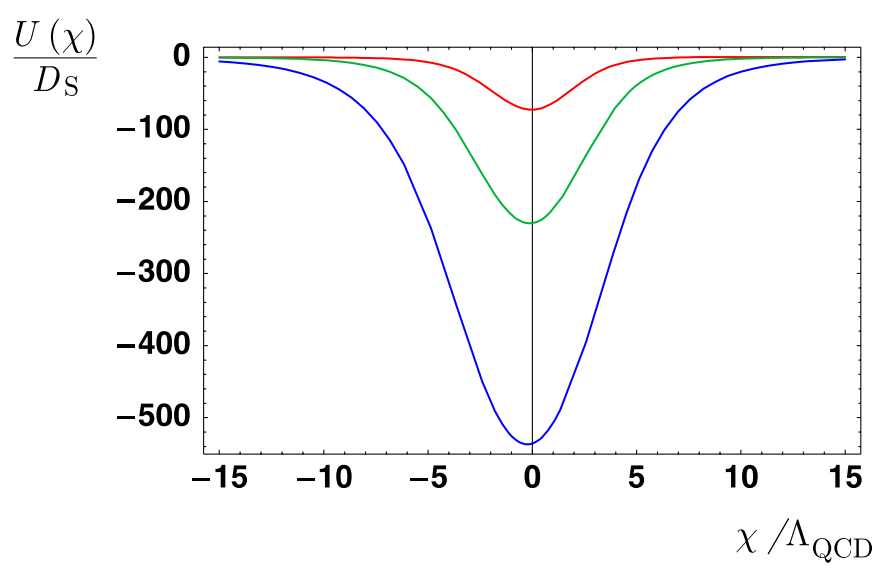

(b)

Fig. 5. Effective potential $U_{\text {inst }}(\sigma, \chi)$ for different fixed $\sigma$. a displays the cases of $\sigma=0,0.06,0.128,0.20 \Lambda_{\mathrm{QCD}}$ in green, black, red and blue, respectively. We observe that for values of $\sigma>\sigma_{\text {crit }} \approx 0.128 \Lambda_{\mathrm{QCD}}$ (red curve) the trivial minimum in the $\chi$ direction becomes the global minimum. b shows the potential for realistic values of $\sigma=1,2,4 \Lambda_{\mathrm{QCD}}$ in red, green and blue, respectively (descending order), clearly demonstrating the absence of an instanton-induced color octet condensate. The parameters are chosen as in Fig. 4 and all numbers refer to units of $\Lambda_{\mathrm{QCD}}$

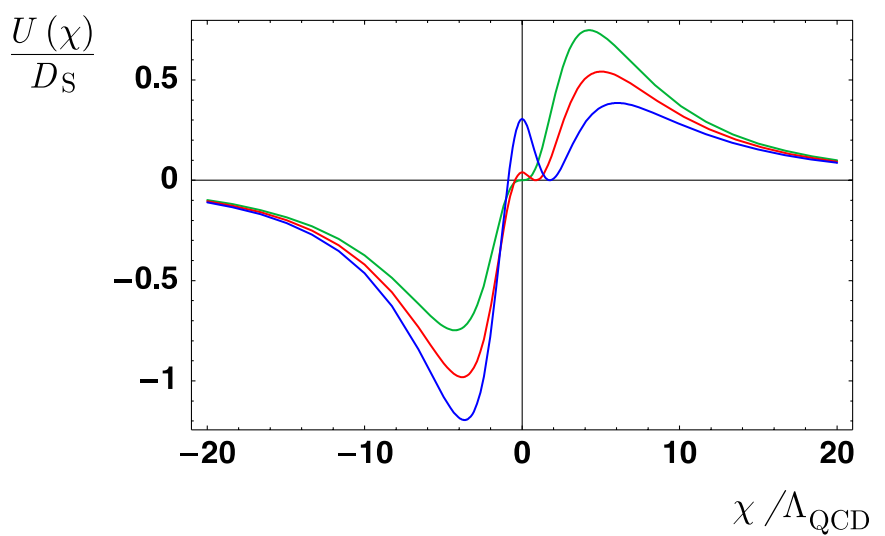

(a)

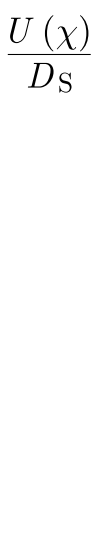

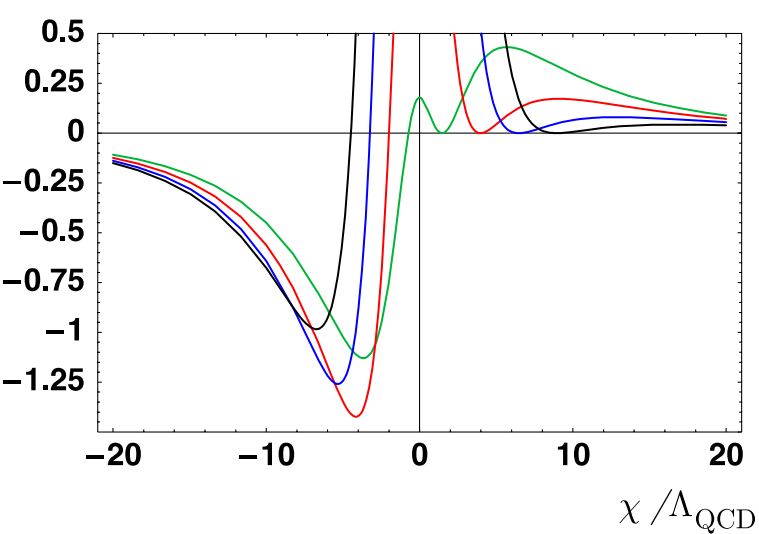

(b)

Fig. 6. $U_{\text {inst }}(\sigma, \chi)$ for negative fixed $\sigma$. In a, we choose $\sigma=0,-0.06,-0.12 \Lambda_{\mathrm{QCD}}$ (green, red and blue). The global minimum then is always at non-vanishing (negative) $\chi$. in $\mathbf{b}$, we depict the behavior for somewhat more negative $\sigma=$ $-0.1,-0.27,-0.44,-0.61 \Lambda_{\mathrm{QCD}}$ (green, red, blue and black). After increasing up to $\sigma \approx-0.27 \Lambda_{\mathrm{QCD}}($ red curve) the minimum in the $\chi$ direction becomes more and more shallow with decreasing $\sigma$. The parameters are chosen as in Fig. 4

by the non-anomalous part (unless the latter is either finetuned or supports an octet condensate itself).

\section{Conclusions}

We have calculated the one-instanton contribution to the effective potential in a background of classical bosonic fields coupled to quarks and gluons. One field, $\sigma$, has the structure of the typical singlet chiral condensate and the other, $\chi$, exhibits a color-flavor-locking structure, as conjectured for the scenario of spontaneous color symmetry breaking in the vacuum. Beyond leading order in the background fields, we have included the effects of the quark masses on the running gauge coupling and the quark de- terminant. In addition, the color octet condensate works as a Higgs field for the gluons, providing for an extra $\chi$-dependent contribution to the classical instanton action. We work in a massive regulator scheme which makes threshold behavior more transparent.

For the realistic case of $N_{\mathrm{f}}=3$ light quark flavors, the instanton potential is unbounded from below for a pure singlet chiral condensate, favoring a non-trivial value of this condensate and chiral symmetry breaking. Although there exists a local extremum with non-vanishing octet contribution, it is rather shallow and thus likely to be washed out by non-instanton effects. Moreover, this extremum has a nearly vanishing value for the chiral condensate, making it phenomenologically unacceptable. As the potential is unbounded from below along the $\sigma$ direction, a global minimum of the instanton potential alone 
is excluded. Other stabilizing effects are typically expected from the $U(1)_{\mathrm{A}}$-preserving sector. Therefore, we have investigated if a color octet condensate is favored at fixed singlet chiral condensate. For realistic positive values of the chiral field $\sigma \sim 1 \Lambda_{\mathrm{QCD}}, \chi=0$ is the global minimum in the color octet direction. For negative $\sigma$ with a similar absolute value, there exists a minimum with non-vanishing octet condensate. However, this minimum is also unnaturally shallow and thus will presumably be washed out by non-instanton effects. Moreover, the negative sign of $\sigma$ is disfavored by the instanton contribution.

Analytic insight into the effective potential can be gained from its asymptotic behavior for large background fields. It is interesting to note that the behavior for very large fields is inherently connected to asymptotic freedom. For instance, large color octet condensates lead to a high-scale decoupling, such that any non-perturbative IR behavior is screened; in this large- $\chi$ direction, the flattening of the potential can directly be related to the perturbative approach to asymptotic freedom. Another extreme example is provided by the case where asymptotic freedom is lost, e.g., owing to too many fermion species; in this case, the potential may go to $-\infty$ faster than the fourth power of the fields, preventing stabilization by a renormalizable non-instanton potential for the bosonic fields. On the other hand, we observe a strong qualitative dependence on the non-perturbative IR behavior of the coupling only for very small fields and more than four light fermion species: here, the increase of the instanton amplitude depends strongly on the infrared details of the gauge coupling and may be changed from the naive $\sigma^{N_{\mathrm{f}}}, \chi^{N_{\mathrm{f}}}$.

In conclusion, barring large higher-order, e.g., multiinstanton, effects, we find that chiral symmetry breaking is supported by instanton effects. On the other hand, the issue of color octet condensation remains inconclusive. In our approximation, we find no evidence that instantons favor a color condensation in the vacuum.

\section{Appendix A: The one-instanton approximation and the instanton gas}

Here we recall the computation of the instanton-induced effective action $\Gamma$ within the dilute-gas approximation. In a dilute and weakly interacting instanton gas, the dominating contribution to the generating functional is the one-instanton and one-anti-instanton contribution. It reads

$$
Z_{1}=-\Omega\left(U_{\mathrm{I}}(\sigma, \chi)+U_{\mathrm{I}}^{*}(\sigma, \chi)\right),
$$

where $\Omega$ denotes the 4 -volume, and $U_{\mathrm{I}}$ is the effective potential corresponding to the product of functional determinants of the fluctuating fields in this background. The anti-instanton contributes with $U_{\mathrm{AI}}(\sigma, \chi)=U_{\mathrm{I}}^{*}(\sigma, \chi)$. Within the dilute-gas approximation, the contribution of the $|n|$-instanton sector is given by $Z_{n}=\frac{\left(Z_{1}\right)^{n}}{n !}$. This leads to the full amplitude

$$
Z=\sum_{n=0}^{\infty} Z_{n}=\sum_{n=0}^{\infty} \frac{\left(Z_{1}\right)^{n}}{n !}=\exp \left(Z_{1}\right)
$$

where we have normalized the zero-instanton amplitude to one. To lowest order in the bosonic background fields, this holds because we included the influence of the fields (masses for the fermions) only for the zero modes. However, in the absence of gauge fields, the Dirac operator has no zero modes. Beyond this approximation, this normalization corresponds to a modification of the non-instanton

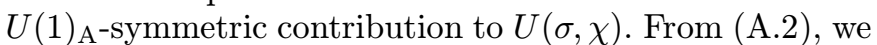
read off the effective action in the classical background of $\sigma, \chi$,

$$
\Gamma=-\ln Z=-Z_{1},
$$

which serves as the starting point of our investigation in the main text.

\section{Appendix B: Different regularization schemes}

In this appendix, we discuss how to switch between regularization schemes. We shall use a scheme which manifestly exhibits the decoupling of massive modes. It has been shown in [7] that topological effects persist within the RG framework used in the present work. This applies, in particular, to the existence of zero modes [7]. Moreover, at leading order one only has to take into account the explicit mass or regulator dependence. This amounts to using the well-known zero modes $[1,2]$.

For non-perturbative problems involving mass threshold effects, as they are induced by the background fields in our case, such schemes are highly advantageous. Following $[1,2,50]$, a change of the renormalization scheme can be understood by comparing two integrals,

$$
I_{1}=\int \frac{\mathrm{d}^{4} q}{(2 \pi)^{4}} \frac{1}{\left(q^{2}+m^{2}\right)^{2}}, \quad I_{2}=\int \frac{\mathrm{d}^{4} q}{(2 \pi)^{4}} \frac{q^{4}}{\left(q^{2}+m^{2}\right)^{4}},
$$

in the different regularization schemes. Here, $I_{1}$ appears in connection with the zero modes and $I_{2}$ with non-zero modes. Therefore, we can easily keep track of these terms. For example, the Pauli-Villars scheme gives

$$
\begin{aligned}
I_{1}^{\mathrm{PV}} & =\int \frac{\mathrm{d}^{4} q}{(2 \pi)^{4}}\left[\frac{1}{\left(q^{2}+m^{2}\right)^{2}}-\frac{1}{\left(q^{2}+\Lambda_{\mathrm{PV}}^{2}\right)^{2}}\right] \\
& =\frac{1}{(4 \pi)^{2}}\left(2 \ln \left(\Lambda_{\mathrm{PV}}\right)-2 \ln (m)\right), \\
I_{2}^{\mathrm{PV}} & =\int \frac{\mathrm{d}^{4} q}{(2 \pi)^{4}}\left[\frac{q^{4}}{\left(q^{2}+m^{2}\right)^{4}}-\frac{q^{4}}{\left(q^{2}+\Lambda_{\mathrm{PV}}^{2}\right)^{4}}\right] \\
& =\frac{1}{(4 \pi)^{2}}\left(2 \ln \left(\Lambda_{\mathrm{PV}}\right)-2 \ln (m)\right)=I_{1}^{\mathrm{PV}},
\end{aligned}
$$


whereas we find in dimensional regularization

$$
\begin{aligned}
I_{1}^{\text {dreg }}= & \mu^{4-n} \int \frac{\mathrm{d}^{n} q}{(2 \pi)^{n}} \frac{1}{\left(q^{2}+m^{2}\right)^{2}} \\
= & \frac{1}{(4 \pi)^{2}}\left[\frac{2}{4-n}+2 \ln (\mu)-2 \ln (m)-\gamma\right. \\
& +\ln (4 \pi)+O(4-n)] \\
= & I_{2}^{\text {dreg }}+\frac{5}{96 \pi^{2}} .
\end{aligned}
$$

Comparing (B.2) and (B.3), the substitutions for a change from Pauli-Villars to dimensional regularization read

$$
\begin{aligned}
& I_{1}: \ln \left(\Lambda_{\mathrm{PV}}\right) \rightarrow \frac{1}{4-n}+\ln (\mu)-\frac{1}{2} \gamma+\frac{1}{2} \ln (4 \pi), \\
& I_{2}: \ln \left(\Lambda_{\mathrm{PV}}\right) \rightarrow \frac{1}{4-n}+\ln (\mu)-\frac{1}{2} \gamma+\frac{1}{2} \ln (4 \pi)-\frac{5}{12} .
\end{aligned}
$$

Using (B.4), it is easy to check that, starting from (9) and (11), we can obtain the corresponding result in dimensional regularization as given, e.g., in $[1,2]$.

Both schemes discussed so far are mass independent. This originates from the fact that for fixed cutoff $\mu$ or fixed dimensionality $4-n$, the integrals $I^{\mathrm{PV}}$ and $I^{\text {dreg }}$ do not vanish in the limit $m \rightarrow \infty$. A mass-dependent regularization scheme should implement this decoupling: massive modes should not contribute to physics below the mass threshold. For $m \gg \mu$ the integrals $I_{1}$ and $I_{2}$ should become small and vanish in the above limit $\mu$ fixed, $m \rightarrow \infty$. This can be implemented by defining $I_{1}^{\mathrm{RG}} \stackrel{!}{=} I_{2}^{\mathrm{RG}}$ with

$$
\begin{aligned}
I_{1}^{\mathrm{RG}} & =\int_{0}^{\Lambda} \mathrm{d} k k^{-1} l\left(\frac{m^{2}}{k^{2}}\right) \\
& =\frac{1}{(4 \pi)^{2}}\left[\ln \left(1+\frac{\Lambda^{2}}{m^{2}}\right)-\frac{2 m^{2} \Lambda^{2}+3 \Lambda^{4}}{2\left(m^{2}+\Lambda^{2}\right)^{2}}\right], \\
l(\omega) & =\frac{1}{(1+\omega)^{3}} .
\end{aligned}
$$

In fact, this is not an arbitrary definition, but one that receives motivation from various sources. First, it is very convenient to have $I_{1}=I_{2}$, since, when changing from the common Pauli-Villars scheme to our scheme it is not necessary to distinguish between the different contributions from $I_{1}$ and $I_{2}$. The main reason, however, is the simple form of the one-loop flow equation for the gauge coupling in (14) which results from this choice. Finally, a deeper reason for the choice is that it corresponds to a simplified version of a typical functional RG scheme regularization (for more details see, e.g. [37]). Indeed,

$$
I_{1}^{\mathrm{RG}}=-\int_{0}^{\Lambda} \frac{\mathrm{d} k}{k} \int \frac{\mathrm{d}^{4} q}{(2 \pi)^{4}} \frac{\mathrm{d}}{\mathrm{d} k} R_{k}\left(q^{2}\right) \frac{1}{\left(q^{2}+m^{2}+R_{k}\left(q^{2}\right)\right)^{3}}
$$

is a typical expression for $I_{1}$ when one defines perturbation theory from a flow equation with regulator function $R_{k}\left(q^{2}\right)$. In a consequent RG calculation several different threshold functions similar to $l(\omega)$ appear. For simplicity we put $I_{1}=I_{2}$. This is not an approximation but simply an implicit definition of the related regulator function $R_{k}$. For computations beyond the present qualitative setting we suggest using an optimized regulator $[51,52]$

$$
R_{k}\left(q^{2}\right)=\left(k^{2}-q^{2}\right) \Theta\left(1-\frac{q^{2}}{k^{2}}\right)
$$

and its upgrades suitable for momentum-dependent approximations [25].

From (B.5), it is easy to find the relation between PauliVillars regularization and our scheme:

$$
\begin{aligned}
\ln \left(\Lambda_{\mathrm{PV}}\right) & \rightarrow \ln (m)+\int_{0}^{\Lambda} \frac{\mathrm{d} k}{k} l\left(\frac{m^{2}}{k^{2}}\right) \\
& =\frac{1}{2} \ln \left(m^{2}+\Lambda^{2}\right)-\frac{2 m^{2} \Lambda^{2}+3 \Lambda^{4}}{4\left(m^{2}+\Lambda^{2}\right)^{2}} .
\end{aligned}
$$

We emphasize that (B.8) depends on the cutoff $\Lambda$ as well as on the mass $m$ of the particle in question. For large $m$, the mass acts similar to the cutoff $\Lambda$. This implements the decoupling of heavy modes.

Finally, we exploit the freedom of redefining the coupling constant at one-loop order, such that it absorbs part of the finite changes discussed above,

$$
\frac{8 \pi^{2}}{g_{\overline{\mathrm{S}}}^{2}(\rho)}=\frac{8 \pi^{2}}{g_{\mathrm{S}}^{2}(\rho)}+C_{\mathrm{S} \overline{\mathrm{S}}}
$$

modifying the perturbative expression for $g^{2}$ only at order $g^{4}$. This is often used to simplify expressions, e.g., in the transition from MS to $\overline{\mathrm{MS}}$, or to ensure direct comparability between different schemes. We will use this freedom below in Appendix $\mathrm{C}$ to facilitate comparisons between our scheme and the $\overline{\mathrm{MS}}$ scheme in which most results are given in the literature.

\section{Appendix C: Assembling the instanton integral}

In this appendix, we put together all the various pieces of the instanton size $\rho$ integral, taking care of our massdependent regularization scheme. At fixed instanton size and using Pauli-Villars regularization, the following functions contribute to the renormalized integrand:

$$
\begin{aligned}
f_{\mathrm{cl}}= & \exp \left(-S_{\mathrm{cl}}\right)=\exp \left(-\frac{8 \pi^{2}}{g^{2}(\mu)}\right), \\
f_{\text {non-zero }}= & \exp \left(-\frac{1}{3} N_{\mathrm{c}} \ln (\mu \rho)-\alpha(1)\right. \\
& \left.+\frac{N_{\mathrm{f}}}{3} \ln (\mu \rho)+2 N_{\mathrm{f}} \alpha\left(\frac{1}{2}\right)\right), \\
f_{\text {gauge }}= & \frac{4}{\rho^{5}}\left(\frac{4 \pi}{g^{2}(\mu)}\right)^{2 N_{\mathrm{c}}}(\mu \rho)^{4 N_{\mathrm{c}}}, \\
f_{\text {fermion }}= & \frac{M^{N_{\mathrm{f}}}}{\mu^{N_{\mathrm{f}}}}, \\
N= & \frac{4}{\pi^{2}} \frac{\pi^{2\left(N_{\mathrm{c}}-1\right)}}{\left(N_{\mathrm{c}}-1\right) !\left(N_{\mathrm{c}}-2\right) !},
\end{aligned}
$$


where $M^{N_{\mathrm{f}}}$ represents $V(\sigma, \chi)$ as defined in (11) and reduces to $m^{N_{\mathrm{f}}}$ if the bosonic sources only lead to a simple mass term $m$.

Here $f_{\mathrm{cl}}$ is the contribution from the classical action, $f_{\text {non-zero }}$ summarizes all effects from the non-zero modes, $f_{\text {gauge }}$ is the contribution from the gauge and $f_{\text {fermion }}$ from the fermion zero modes. $N$ collects some normalization factors and the group averaging. Combining all these contributions, we find the well-known result

$$
\begin{aligned}
N \prod_{x} f_{x}= & D_{\mathrm{PV}} \rho^{-5+N_{\mathrm{f}}}\left(\frac{8 \pi^{2}}{g^{2}(\mu)}\right) \\
& \times \exp \left(-\frac{8 \pi^{2}}{g^{2}(\mu)}+\beta_{0} \ln (\mu \rho)\right) \\
= & D_{\mathrm{PV}} \rho^{-5+N_{\mathrm{f}}}\left(\frac{8 \pi^{2}}{g^{2}(\mu)}\right) \exp \left(-\frac{8 \pi^{2}}{g_{\mathrm{PV}}^{2}(\rho)}\right),
\end{aligned}
$$

where we have used the one-loop relation between $g^{2}(\mu)$ and $g^{2}(\rho)$ in the last step, and $D$ is given in the PauliVillars scheme as

$$
\begin{aligned}
D_{\mathrm{PV}} & =\exp \left(-\alpha(1)-2\left(N_{\mathrm{c}}-2\right) \alpha\left(\frac{1}{2}\right)+2 N_{\mathrm{f}} \alpha\left(\frac{1}{2}\right)\right) \\
& =1.1506 \\
\alpha\left(\frac{1}{2}\right) & =2 R-\frac{1}{6} \ln (2)-\frac{17}{72}=0.1459, \\
\alpha(1) & =8 R=\frac{1}{3} \ln (2)-\frac{16}{9}=0.4433, \\
R & =\frac{1}{12}(\ln (2 \pi)+\gamma)+\frac{1}{2 \pi^{2}} \sum_{s=2}^{\infty} \frac{\ln (s)}{s^{2}} \\
& =0.2488 .
\end{aligned}
$$

We point out that we still have $g^{2}(\mu)$ in the prefactor of the exponential, which is an artifact of the one-loop calculation. This will be rectified by higher-loop orders where the 'bare' $g^{2}(\mu)$ in the prefactor is replaced by its running counterpart evaluated at the scale $\rho$ (also at one-loop order less than the corresponding one in the exponential). Since replacing $g^{2}(\mu) \rightarrow g^{2}(\rho)$ is the main effect of higher-loop orders (apart from possible changes in the factor $D$ ), we account for these prefactors as well as for the term in the exponential by hand without further calculation. In this way, we already arrive at (9) and (12) (at least naively in the Pauli-Villars scheme),

$$
N \prod_{x}=D_{\mathrm{PV}} \rho^{-5+N_{\mathrm{f}}}\left(\frac{8 \pi^{2}}{g_{\mathrm{PV}}^{2}(\rho)}\right) \exp \left(-\frac{8 \pi^{2}}{g_{\mathrm{PV}}^{2}(\rho)}\right) .
$$

The final task now is to change from the mass-independent Pauli-Villars scheme to our mass-dependent RG scheme. Starting from (C.8), this is immediately done using (B.8), resulting in an additional multiplicative factor,

$$
\begin{aligned}
\mathrm{RG}\left(M_{g} \rho, m_{f} \rho\right) & =\exp \left(\frac{11}{3} N_{\mathrm{c}} H\left(M_{g} \rho\right)-\frac{2}{3} N_{\mathrm{f}} H\left(m_{f} \rho\right)\right), \\
H(x) & =\frac{1}{2} \ln \left(1+x^{2}\right)-\frac{3+2 x^{2}}{4\left(1+x^{2}\right)^{2}},
\end{aligned}
$$

where $M_{g}$ and $m_{f}$ are the gauge boson and fermion masses, respectively.

Finally, we make a last change and define a modified $\overline{\mathrm{RG}}$ scheme via (B.9) with

$$
C_{\mathrm{RG} \overline{\mathrm{RG}}}=-\ln \left(\frac{\mathrm{RG}(0,0) D_{\mathrm{PV}}}{D_{\overline{\mathrm{MS}}}}\right),
$$

where $D_{\overline{\mathrm{MS}}}$ is defined in (10). By construction, this establishes that our coupling constant is equal to the one-loop $\overline{\mathrm{MS}}$ coupling, also including the constant in the instanton integral. But most importantly, we have not absorbed the mass-dependent contributions. Therefore, our scheme is still mass-dependent and provides for decoupling of heavy modes.

\section{Appendix D: Calculation of the zero-mode part}

The Dirac operator $\not D$ in the background of an instanton has $N_{\mathrm{f}}=3$ zero modes, being flavor copies of a fundamental zero mode. We show that in leading order

$$
\begin{aligned}
\zeta_{\mathrm{z}}(\rho, \sigma, \chi) & =\left\langle\operatorname{det}_{\text {flavor }}\left\langle\psi_{0}(a, i)\left|M_{\psi, i j}\right| \psi_{0}(b, j)\right\rangle\right\rangle_{S U(3)} \\
& =:-\rho^{N_{\mathrm{f}}} V(\sigma, \chi),
\end{aligned}
$$

where

$$
\left(M_{\psi, i j}\right)_{\alpha \beta}=\left(\left(-\not D_{i j}\right)_{\alpha \beta}+\left(\sigma \delta_{i j}+\chi_{i j}\right) \delta_{\alpha \beta}\right),
$$

with mass matrix $\sigma+\chi$, where $\chi$ introduces color-flavor mixing. The eigenvalues of $M_{\psi}$ are $\lambda_{n}(\sigma, \chi)$ with eigenfunctions $\psi_{n}(\sigma, \chi)$. The color-flavor mixing term $\chi$ does not commute with $\not D$, and the $\psi_{n}$ are not eigenfunctions of $\not D$ for $\chi \neq 0$, leaving (D.1) a non-trivial identity. The mass matrix reads more explicitly

$$
\begin{aligned}
& \left(\sigma_{a b} \delta_{i j}+\chi_{a b, i j}\right) \delta_{\alpha \beta} \\
& =\left[\sigma \delta_{a b} \delta_{i j}+\frac{1}{\sqrt{6}} \chi\left(\delta_{a i} \delta_{b j}-\frac{1}{3} \delta_{a b} \delta_{i j}\right)\right] \delta_{\alpha \beta},
\end{aligned}
$$

where we have already absorbed the Yukawa couplings into the fields and used the color-flavor structure (2) for the condensates. For clarity we have explicitly written out the spin indices $\alpha, \beta$. Using the group averages in (D.1) $[3,53]$, we arrive at

$$
\zeta_{\mathrm{z}}(\rho, \sigma, \chi)=\rho^{N_{\mathrm{f}}}\left(\sigma+\frac{1}{6 \sqrt{6}} \chi\right)^{2}\left(\sigma-\frac{1}{3 \sqrt{6}} \chi\right) .
$$

It is left to prove (D.1). With trivial flavor structure, $\chi_{i j}=0$, the determinant factorizes trivially,

$$
\operatorname{det} M_{\psi}=\zeta_{\mathrm{z}}(\rho, \sigma, \chi) \operatorname{det}^{\prime} M_{\psi},
$$

where $\operatorname{det}^{\prime}$ stands for the determinant on the non-zero mode space. For non-vanishing $\chi$ (D.5) holds up to terms $\chi^{N_{\mathrm{f}}}$. This is shown in an expansion about the determinant 
of $-\not D+\sigma$ with eigenvalues $\lambda_{n}(\sigma, 0)=\lambda_{n}(0,0)+\sigma$ and eigenfunctions $\psi(\sigma, 0)=\psi_{n}(0,0)$. We label the zero modes of $\not D$ with $\psi_{n_{0}}, n_{0}=1,2,3$ with eigenvalues $\lambda_{n_{0}}(s=0)=\sigma$. There is no term linear in $\chi$ as the only invariant is $\operatorname{tr}_{\text {flavor }} \chi=0$. The quadratic term has the structure $\sigma u_{2}(\sigma) \chi^{2}$ with finite limit $u(0)$. It is evaluated as

$$
\begin{aligned}
\left.\frac{1}{2} \partial_{s}^{2} \operatorname{det}(-\not D+\sigma+s \chi)\right|_{s=0}= & \frac{1}{2} \sum_{n}\left(\partial_{s}^{2} \lambda_{n}\right) \prod_{m \neq n} \lambda_{m} \\
& +\sum_{m<n}\left(\partial_{s} \lambda_{n}\right)\left(\partial_{s} \lambda_{m}\right) \prod_{l \neq n, m} \lambda_{l},
\end{aligned}
$$

where the limit $s=0$ on the right-hand side of (D.6) is understood. The term proportional to $\partial_{s}^{2} \lambda_{n}$ has the coefficient $\prod_{m \neq n} \lambda_{m}$ containing at least two of the eigenvalues $\lambda_{n_{0}}(s=0)=\sigma$ of the zero modes $\psi_{n_{0}}$. Hence it only contributes to sub-leading terms like $\rho \sigma^{2} \chi^{2}$. The term proportional to $\left(\partial_{s} \lambda\right)^{2}$ has, apart from sub-leading terms, one contribution proportional to $\prod_{l \neq n_{0}, m_{0}} \lambda_{l}$ removing two zero modes from the product. Thus we have

$$
\begin{aligned}
u_{2}(\sigma) \sigma^{N_{\mathrm{f}}-2} \chi^{2}= & \sigma^{N_{\mathrm{f}}-2} \sum_{m_{0}<n_{0}}\left(\partial_{s} \lambda_{n_{0}}\right)\left(\partial_{s} \lambda_{m_{0}}\right) \operatorname{det}^{\prime} M_{\psi} \\
& +O\left(\sigma^{2} \chi^{2}\right)
\end{aligned}
$$

where $\operatorname{det}^{\prime} M_{\psi}=\prod_{l}^{\prime} \lambda_{l}$, and the primed product involves only the non-zero eigenvalues of $\not D$. The $s$ derivatives in (D.7) follow:

$$
\begin{aligned}
\partial_{s} \lambda_{n}(s=0) & =\partial_{s}\left\langle\psi_{n}|(-\not D+\sigma+s \chi)| \psi_{n}\right\rangle \\
& =\left\langle\psi_{n}|\chi| \psi_{n}\right\rangle+\lambda_{n} \partial_{s}\left\langle\psi_{n} \mid \psi_{n}\right\rangle=\left\langle\psi_{n}|\chi| \psi_{n}\right\rangle
\end{aligned}
$$

where we have used $\left\langle\partial_{s} \psi_{n}|(-\not D+\sigma)| \psi_{n}\right\rangle=$ $\left\langle\partial_{s} \psi_{n} \mid \psi_{n}\right\rangle \lambda_{n}$ and $\left\langle\psi_{n}|(-\not D+\sigma)| \partial_{s} \psi_{n}\right\rangle=\lambda_{n}\left\langle\psi_{n} \mid \partial_{s} \psi_{n}\right\rangle$. We arrive at

$$
u_{2}(0) \chi^{2}=\sum_{m_{0}<n_{0}}\left\langle\psi_{n_{0}}|\chi| \psi_{n_{0}}\right\rangle\left\langle\psi_{m_{0}}|\chi| \psi_{m_{0}}\right\rangle \operatorname{det}^{\prime} M_{\psi}
$$

Equation (D.9) extends to the general $u_{n}(0) \chi^{n}$ with $n \leq$ $N_{\mathrm{f}}$. We are specifically interested in $N_{\mathrm{f}}=3$ with the remaining cubic term $u_{3}(\sigma) \sigma^{N_{\mathrm{f}}-3} \chi^{3}=u_{3}(\sigma) \chi^{3}$, in leading order,

$$
u_{3}(0) \chi^{3}=\left(\prod_{n_{0}}\left\langle\psi_{n_{0}}|\chi| \psi_{n_{0}}\right\rangle\right) \operatorname{det}^{\prime} M_{\psi} .
$$

This proves (D.1). We can also directly use (D.9) and (D.10) to compute the $\chi^{2}$ and $\chi^{3}$ coefficients as group averages $\left\langle u_{2}\right\rangle_{S U(3)},\left\langle u_{3}\right\rangle_{S U(3)}$, with the help of [53]. We arrive at

$$
\begin{aligned}
& \frac{3}{\chi^{2}}\left\langle\prod_{n_{0}=1}^{2}\left\langle\psi_{n_{0}}|\chi| \psi_{n_{0}}\right\rangle\right\rangle_{S U(3)}=\frac{1}{72} \\
& \frac{1}{\chi^{3}}\left\langle\prod_{n_{0}=1}^{3}\left\langle\psi_{n_{0}}|\chi| \psi_{n_{0}}\right\rangle\right\rangle_{S U(3)}=\frac{1}{648 \sqrt{6}}
\end{aligned}
$$

leading to (12).

\section{Appendix E: IR running coupling effects in the lowest-order approximation}

Here we demonstrate that the qualitative features of the instanton-induced effective potential to lowest order in the scalar condensates is largely independent of the behavior of the running coupling. This can be deduced from a study of the $\rho$ integration in (12). For constant $g^{2}(\rho)=g^{2}$ and $N_{\mathrm{f}}<4$, the $\rho$ integral is infrared $(\rho \rightarrow \infty)$ convergent, but it has a (naive) UV divergence. The one-loop

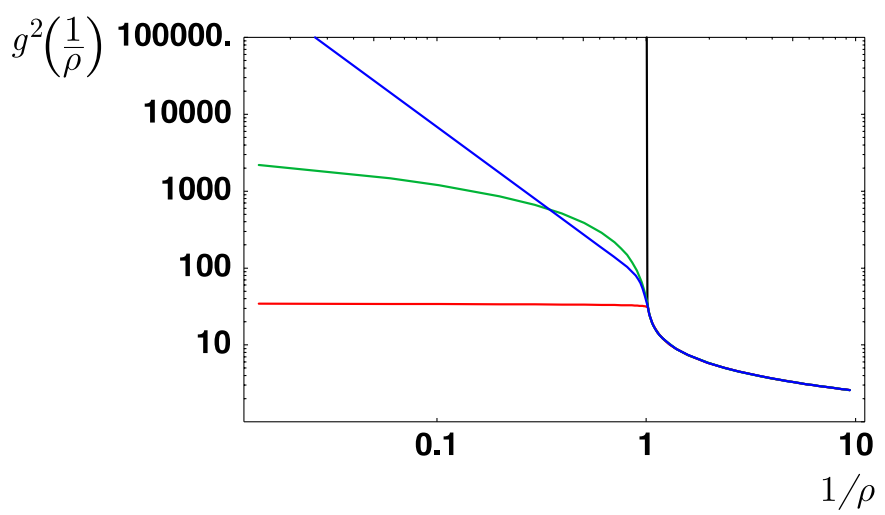

(a)
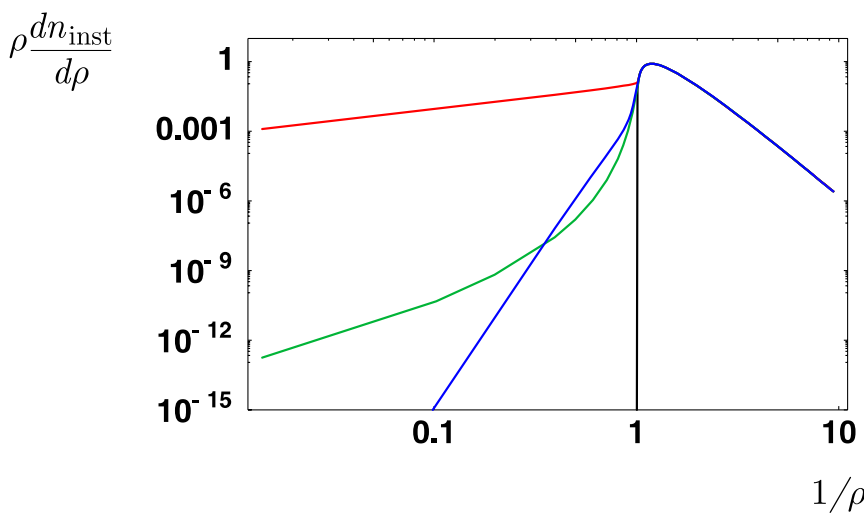

(b)

Fig. 7. Instanton density $\mathbf{b}$ for various types of infrared behavior for the gauge coupling $\mathbf{a}$. Red: $g \sim$ const., green: $g \sim \ln (\rho)$, blue: $g \sim \rho^{2}$, black: one loop behavior with $g=\infty$ for $\frac{1}{\rho}<\Lambda_{\mathrm{QCD}}$. Strong non-perturbative behavior is modeled to set in at roughly $g^{2} \approx$ 30. We note that the integral over the instanton size remains finite. More quantitatively, this holds if the plotted quantity in $\mathbf{b}$ vanishes faster than $\frac{1}{\rho^{\epsilon}}$ with $\epsilon>0$ in the infrared. Most importantly, the constant $\zeta$ occurring in our lowest order approximation to the effective potential, (12), remains a finite number 
running removes this UV divergence, because of asymptotic freedom. Then the integration kernel behaves as $\sim$ $\rho^{\frac{11}{3} N_{\mathrm{c}}+\frac{1}{3} N_{\mathrm{f}}-5}(\ln (\rho))^{2 N_{\mathrm{c}}}$ for small $\rho$, rendering the integral convergent in this regime. In the infrared, the situation is less clear, since one-loop running is certainly not a valid approximation for the gauge coupling. Nevertheless, the restrictions on the behavior of $g^{2}(\rho)$ for $\rho \rightarrow \infty$ are rather mild. Indeed, for positive and well-defined $g^{2}(\rho)$ there are no restrictions at all for $N_{\mathrm{f}}<4$ (massless flavors). We can even allow for a diverging coupling at a finite infrared scale $\rho_{\text {div }}$. In this case, it is reasonable to assume that the coupling remains infinite for even larger distance scales, such that the integrand remains exactly zero for all $\rho>$ $\rho_{\text {div }}$. In Fig. 7 we plot the integrand for running couplings with different infrared behavior. It is our main conclusion that all reasonable forms for the running coupling in the infrared imply a finite constant $\zeta$ in (12).

Acknowledgements. The authors are grateful to G.V. Dunne, H. Min, and A. Ringwald for discussions. HG, JJ, and JMP acknowledge support by the DFG under Gi 328/1-3 (EmmyNoether program).

\section{References}

1. G. 't Hooft, Phys. Rev. D 14, 3432 (1976)

2. G. 't Hooft, Phys. Rev. D 18, 2199 (1978) [Erratum]

3. M.A. Shifman, A.I. Vainshtein, V.I. Zakharov, Nucl. Phys. B 163, 46 (1980)

4. C.G. Callan, R.F. Dashen, D.J. Gross, Phys. Rev. D 17, 2717 (1978)

5. E.V. Shuryak, Nucl. Phys. B 203, 140 (1982)

6. T. Schaefer, E.V. Shuryak, Rev. Mod. Phys. 70, 323 (1998) [hep-ph/9610451]

7. J.M. Pawlowski, Phys. Rev. D 58, 045011 (1998) [hepth/9605037]

8. C. Wetterich, Phys. Lett. B 525, 277 (2002) [hep-ph/ 0011076]

9. E. Meggiolaro, C. Wetterich, Nucl. Phys. B 606, 337 (2001) [hep-ph/0012081]

10. D. Diakonov, V.Y. Petrov, Phys. Lett. B 147, 351 (1984)

11. D. Diakonov, V.Y. Petrov, Nucl. Phys. B 272, 457 (1986)

12. G.W. Carter, D. Diakonov, hep-ph/9905465

13. D. Bailin, A. Love, Phys. Rep. 107, 325 (1984)

14. M.G. Alford, K. Rajagopal, F. Wilczek, Phys. Lett. B 422, 247 (1998) [hep-ph/9711395]

15. R. Rapp, T. Schaefer, E.V. Shuryak, M. Velkovsky, Phys. Rev. Lett. 81, 53 (1998) [hep-ph/9711396]

16. M.G. Alford, K. Rajagopal, F. Wilczek, Nucl. Phys. B 537, 443 (1999) [hep-ph/9804403]

17. J. Berges, K. Rajagopal, Nucl. Phys. B 538, 215 (1999) [hep-ph/9804233]

18. C. Wetterich, Phys. Lett. B 462, 164 (1999) [hep-th/ 9906062]

19. C. Wetterich, Phys. Rev. D 64, 036003 (2001) [hep$\mathrm{ph} / 0008150]$
20. J. Jaeckel, C. Wetterich, Nucl. Phys. A 733, 113 (2004) [hep-ph/0309101]

21. J. Jaeckel, C. Wetterich, Phys. Rev. D 68, 025020 (2003) [hep-ph/0207094]

22. D.F. Litim, J.M. Pawlowski, in: The Exact Renormalization Group, ed. by A. Krasnitz et al. (World Scientific, Singapore, 1999), p. 168 [hep-th/9901063]

23. J. Berges, N. Tetradis, C. Wetterich, Phys. Rep. 363, 223 (2002) [hep-ph/0005122]

24. J. Polonyi, Eur. Phys. J. C 1, 1 (2004) [hep-th/ 0110026]

25. J.M. Pawlowski, hep-th/0512261

26. U. Ellwanger, M. Hirsch, A. Weber, Z. Phys. C 69, 687 (1996) [hep-th/9506019]

27. J.M. Pawlowski, D.F. Litim, S. Nedelko, L. von Smekal, Phys. Rev. Lett. 93, 152002 (2004) [hep-th/0312324]

28. C.S. Fischer, H. Gies, JHEP 0410, 048 (2004) [hep-ph/ 0408089]

29. J.P. Blaizot, R. Mendez Galain, N. Wschebor, Phys. Lett. B 632, 571 (2006) [hep-th/0503103]

30. M. Reuter, C. Wetterich, Nucl. Phys. B 417, 181 (1994)

31. F. Freire, D.F. Litim, J.M. Pawlowski, Phys. Lett. B 495, 256 (2000) [hep-th/0009110]

32. H. Gies, Phys. Rev. D 66, 025006 (2002) [hep-th/0202207]

33. H. Gies, C. Wetterich, Phys. Rev. D 65, 065001 (2002) [hep-th/0107221]

34. H. Gies, C. Wetterich, Phys. Rev. D 69, 025001 (2004) [hep-th/0209183]

35. H. Gies, J. Jaeckel, Eur. Phys. J. C 46, 433 (2006) [hepph/0507171]

36. C. Wetterich, cond-mat/0208361

37. J. Jaeckel, hep-ph/0309090

38. T. Schaefer, F. Wilczek, Phys. Rev. Lett. 82, 3956 (1999) [hep-ph/9811473]

39. C. Wetterich, Eur. Phys. J. C 18, 577 (2001) [hep-ph/ 9908514]

40. C. Wetterich, AIP Conf. Proc. 739, 123 (2005) [hep$\mathrm{ph} / 0410057]$

41. G. 't Hooft, Phys. Rep. 142, 357 (1986)

42. C.W. Bernard, Phys. Rev. D 19, 3013 (1979)

43. M. Luscher, Nucl. Phys. B 205, 483 (1982)

44. A. Hasenfratz, P. Hasenfratz, Nucl. Phys. B 193, 210 (1981)

45. A. Ringwald, F. Schrempp, Phys. Lett. B 459, 249 (1999) [hep-lat/9903039]

46. G.V. Dunne, J. Hur, C. Lee, H. Min, Phys. Lett. B 600, 302 (2004) [hep-th/0407222]

47. G.V. Dunne, J. Hur, C. Lee, H. Min, Phys. Rev. Lett. 94, 072001 (2005) [hep-th/0410190]

48. R.D. Carlitz, D.B. Creamer, Ann. Phys. 118, 429 (1979)

49. O.K. Kwon, C.K. Lee, H. Min, hep-th/0003040

50. G. 't Hooft, Nucl. Phys. B 62, 444 (1973)

51. D.F. Litim, Phys. Lett. B 486, 92 (2000) [hep-th/0005245]

52. D.F. Litim, Phys. Rev. D 64, 105007 (2001) [hep-th/ 0103195]

53. M. Creutz, Quarks Gluons and Lattices (Cambridge University Press, Cambridge, 1983) 